\title{
Improving the Network Lifetime for WSNs using Multiple Data Collectors
}

\author{
Rupinder Kaur \\ Student, Dept of CSE \\ Amritsar College of Engineeringv \& Technology \\ PTU, Jalandhar ,Punjab.
}

\author{
Tanupreet Singh, Ph.D. \\ Professor and HOD, Dept of ECE \\ Amritsar College of Engineering \& Technology \\ PTU, Jalandhar, Punjab
}

\begin{abstract}
Energy is the main component of sensor nodes. IEESSC used sink mobility to increase the network lifetime. IEESSC also leads to uneven cluster. EEEGBC proposed algorithm uses grid based method to make same size clusters and it also used the concept of multiple sink to increase the network lifetime. It removes the idea of re-clustering and energy is saved that use for re-clustering. The simulation analysis are done based on three parameters: Network Lifetime, Stability Period and Middle Node Death.
\end{abstract}

\section{Keywords}

LEACH, EESSC, IEESSC, SPIN, EEEGBC, WSN, CH.

\section{INTRODUCTION}

Wireless Sensor Networks were firstly planned to smooth the progress of military operations however its applications has been extended to health, traffic, and many other consumer and industrial areas. A wireless sensor network is a group of specialized transducers with a communications infrastructure for monitoring and recording conditions at diverse locations. Commonly monitored parameters are temperature, humidity, pressure, wind direction and speed, illumination intensity, vibration intensity, sound intensity, power-line voltage, chemical concentrations, pollutant levels and vital body functions. A sensor network consists of multiple detection stations called sensor nodes, each of which is small, lightweight and portable. Every sensor node is equipped with a transducer, microcomputer, transceiver and power source. The transducer generates electrical signals based on sensed physical effects and phenomena. The microcomputer processes and stores the sensor output. The transceiver receives commands from a central computer and transmits data to that computer. The power for each sensor node is derived from a battery.

\section{RELATED WORK}

A new routing algorithm ADADR [21] has been proposed based on the concept of potential in the discipline of physics. The main objective of this paper has been to get better the degree of data aggregation and minimize ANT. Packet attribute is taken as the main element for data aggregation. The packet delivery to the last node has assured by the hybrid potential field. A pheromone value has used to differentiate the packets with the same attribute to make the packets convergent. The advantage of this routing algorithm has been that it has more energy efficient for data aggregation in WSNs. It has been also simple and scalable based potential based dynamic routing scheme.

Various routing protocols [25] has been studied and categorized in terms of Flat based Routing, Hierarchical based
Routing and Location based Routing. These algorithms has been analyzed and compared in terms of energy, data aggregation, delay, throughput, bandwidth, and link cost and network lifetime. Energy awareness has the current issue in the wireless sensor network. A protocol should be proposed to satisfy all the conditions like increase the throughput, maximize the lifetime, reliability of data and high packet delivery ratio.

A new routing protocol IEESSC [5] has been proposed. EESSC has improved by using less number of cluster formation.A new protocol used the concept of sink mobility which reduced communication cost. Sink changed its position randomly after every round. This would lead to uniform energy consumption and increased network lifetime. The proposed algorithm has been compared with LEACH and EESSC protocol on the basis of different performance metrics $\backslash 1 / /$ like network lifetime, middle node death and stability. The proposed scheme has shown better performance over existing protocol. IEESSC helped in consuming energy uniformly and improving the network lifetime.

Various LEACH [16] enhancement protocols have been analyzed and compared with each other. Various protocols has been designed to solve the problem of LEACH protocol like delay, stability, localization and mobility of nodes, uniform distribution of $\mathrm{CHs}$, multi-hop routing and optimal $\mathrm{CH}$ selection. LEACH protocol has been enhanced by improvement in three parameters: Modified $\mathrm{CH}$ selection algorithm, Energy aware algorithms, Optimized in $\mathrm{CH}$ selection. Modified LEACH algorithms have been compared on the basis of Communication pattern, Energy Efficiency, Advantages and Limitations. LEACH algorithm should be used in multi-hop communication pattern and election of cluster heads in future.

A new routing protocol LEACH-R [9] is proposed which is an improvement of LEACH protocol. This protocol selected the cluster head on the basis of residual energy of nodes. This would lead to less possibility of selecting the low energy node. A relay node was selected between base station and other cluster heads based on the residual energy and distance from base station. LEACH-R protocol performance were analyzed and compared with LEACH protocol. The simulations of these protocols were done on MATLAB environment and fourth generation language. The simulation result showed that LEACH-R protocol increased the network lifetime and reduced the overall network energy consumption.

Various routing protocols [3] has been discussed on the basis of data-centric routing, hierarchical routing, and location based routing. SPIN, ACQUIRE, EEABR data centric algorithms have been discussed in which sink nodes send 
queries to certain regions to collect the data and attributebased naming has used to specify the property of data. Hierarchical based routing protocols are divided into basically two approaches: Clustering approach and Tree based approach. LEACH and HEED protocols has discussed from clustering approach. PEGASIS has discussed fro tree based routing approach.GAF and GEAR Location-based routing protocols has discussed. All the routing protocols have same objectives to maximize the lifetime of network. This paper concluded that energy efficient routing protocol for mobile wireless sensor networks should be proposed in future.

A new routing protocol [23] based on hierarchical and cluster has been proposed. Sink and sensor node was movable in this protocol. This protocol worked in two phases: Setup Phase and Data Forwarding Phase. Initially entire sensor environment has been divided into clusters. In each cluster, there were two cluster head, one gateway node, and ordinary sensor nodes. The simulation of protocol has been implemented in NS2 simulator and result was compared with Cluster based routing protocol. The performance of proposed routing protocol has been considered in terms of Throughput, Network Life Time, average energy consumption and Average Control Overhead. The energy efficiency and performance of proposed protocol has been better than CBR protocol.

An energy efficient routing protocol Equalized Cluster Head Election Routing Protocol (ECHERP) [28] has been proposed. The current and future estimated remaining energy of the nodes are considered to make the cluster head in every round. The main purpose of using energy for making cluster head is to increase the lifetime of a network. At every round, the Gaussian elimination algorithm used to calculate the energy consumed in the network and reduce the overall energy consumption in the network. ECHERP also used the multihop routing scheme to force the data send backward to the base station. ECHERP is analyzed and compared with earlier protocol like LEACH, PEGASIS and BCDCP through simulation in terms of last node depletion time and first node depletion time. This algorithm could be expanded in terms of QoS and time constrictions.

An energy-efficient competitive clustering algorithm[11] has been proposed for wireless sensor networks using a controlled mobile sink. Sensor nodes has effectively organize by clustering algorithm and controlled mobile sink lead to lessen the problem of energy holes and hot spot problem.NP-hard problem has used to select the optimal moving trajectory for sink nodes. This algorithm [11] has changed the cluster head in each round and selected the cluster head based on their range and remaining energy. Sink has mobile in nature as compares to fixed nature. The sink node has moved in predefined path at certain speed and stayed at park position to collect the data packets. The simulation has done on MATLAB environment to analyze the performance of an energy-efficient competitive clustering algorithm and compared with predefined algorithm LEACH. The simulation result has showed better performance than LEACH algorithm.

AOMDV has been used to transfer the data [30] from sink node to destination node by using multiple paths. In this protocol, sink node was static and all other nodes were movable in nature. Due to static nature of sink, energy consumption was reduced because of less overhead. The simulation of energy efficient routing protocol was done on NS2 simulator in the Linux environment. The performance of this protocol was analyzed and compared with Ad Hoc on Demand Distance Vector Routing Protocol in terms of energy consumption, throughput, packet delivery ratio, total dropped packet, and average end to end delay. The result of simulation proved that it consumed less energy and given high throughput.

In this paper has proposed An Energy Efficient Level Based Clustering Routing Protocol[19] for Wireless Sensor Networks for increasing the lifetime of networks. The entire network has divided into annular rings based upon various power levels at base station and each ring having various sensor nodes. In each cluster head has been selected based upon the value of remaining energy and distance from the base station. The mathematical formulae has also used for selecting the cluster head. The developed model has simulated on MATLAB environment. The result of this protocol was analyzed and compared with LEACH protocol. The simulation result showed that it reduced the energy consumption of $\mathrm{CH}$, reduced the number of clusters, and increased the lifetime of network.

A distributed algorithm [8] with multiple dynamic sink is proposed to improve the lifetime of a network and to minimize the transmission delay form source node to destination sink nodes. Multiple dynamic sinks are used to aggregate the data and receive the data from all sensors. A linear programming model is proposed to reduce the overall energy consumption of a network. MATLAB simulation of this protocol proved that the lifetime of a network is increased. Multiple dynamic sinks reduced the delay in the network and increased the cost of multiple dynamic sinks at a very small rate in comparison with single mobile and static sink. This algorithm could be expanded for reliability and recovery factors in large wireless sensor networks.

The problem of imbalanced energy consumption by nodes has been removed by proposing a cluster based routing protocol with nonuniform node distribution. A competition range used by EDAC [10]to make cluster head .Cluster head has chosen from neighbor nodes list which have highest residual energy. Cluster head send the Hello messages to nodes in competition range for making the equal size of clusters. Inter cluster communication and intra cluster communication used for balancing the energy consumption in dense areas. Simulation has performed on NS2 simulator to see the network lifetime in randomly deployed nodes and nonuniformly distributed nodes. This algorithm has compared with LEACH protocol. It has increased the network lifetime in both scenarios.

Routing protocols has been surveyed based on data communication approach. A new simple and effective scheme [27] is proposed. Energy efficiency and node failure are the two main factors which are considered in this algorithm. In this scheme, cluster coordinators are used to calculate intercluster manage and transmit the data between these clusters. This increased the network lifetime and balanced the energy consumption in the network. This scheme should be used for mobile infrastructure in sensor networks. Inter-cluster communication should be optimized by taking specific measures in future.

A distributed clustering algorithm[4] has been proposed based on the hop distance to the data sink for balancing the node lifetimes and reducing the energy consumption of the network. A simple energy-efficient multihop data collection algorithm has also been proposed and used to estimate the effectiveness of the energy-efficient clustering and determine the end to end consumption of this protocol. Energy-efficient clustering has suitable for energy conservation data collection protocol. The performance of this protocol has been compared 
and analyzed with HEED and UCR algorithms by simulating on MATLAB environment. The network lifetime has increased and balanced energy nodes.

Various challenges and design issues in Wireless Sensor Networks [7] are discussed. Various types of routing protocols are surveyed in terms of different categories like Negotiations based, Multipath based, Query based, Location based, Cluster based, Flat based .Few Protocols are also discussed belonging to these categories. Energy consumption and network lifetime are the main issues in Wireless Sensor Networks routing. Security, Quality of Service and node mobility are the issues which should discussed in future.

A new energy efficient routing protocol Weighted Election Protocol (WEP)[17] has been proposed. Chain routing algorithm with clustering scheme to improve the stable period and energy consumption used in wireless sensor network under heterogeneous environment.WEP has compared with LEACH, SEP , and HEARP. It has better performance than these protocols in terms of network lifetime and stability period, and it has proved by simulation. The drawback of this protocol has that Longley stability period consumed extra energy on setting up heterogeneous environment.

An adaptive clustering strategy[6] has been proposed to elect the cluster heads and next heads in wireless sensor networks. The remaining energy and average energy of each cluster has taken to select the cluster head and next head of each cluster. The cluster head of each cluster has changed dynamically and sensor node with the highest remaining energy has selected to become a cluster head. The simulation of adaptive decentralized re-clustering protocol has done on OMNET++ simulator. The performance of proposed protocol has compared and analyzed with LEACH-C protocol. In single hop communication mode, highest energy consumption due to distance between sensor node and base station. In multihop communication mode, there has highest load of relaying packets on sensor nodes due to closer distance between base station and sensor nodes. This protocol could be extended to find the optimum communication in single hop and multihop clusters.

\section{IEESSC}

Improved energy efficient semi-static routing clustering protocol used clustering for energy saving. Energy is improved by reducing communication cost for re-clustering. This protocol uses semi static sink to reduce the communication cost. Sink changed the position randomly after each round. This leads to uniform energy consumption and increase network lifetime. This scheme used following steps:

1. Initially network is initialized by sending activation packet and every node in a network assumes itself as a cluster.

2. Clusters are formed by using distance and residual energy.

3. Cluster Head are chosen from the cluster with highest energy and it changes after each round.

4. $\mathrm{CH}$ aggregates the data send from the cluster node and send it to the sink.

5. After each round sink changed its position randomly so that energy consumption is uniform.

6. If each round $\mathrm{CH}$ has not sufficient energy, then reclustering is done.

\section{EEEGBC}

Enhanced energy efficient grid based routing clustering protocol also used the clustering method to decrease the communication cost. The clustering is done on the basis of grid. The whole area is divided into grid and the nodes are presented in one grid represents one cluster. EEEGBC used subsink in each cluster instead of cluster head. The grid based clustering removes uneven cluster problem and it also removes the concept of re-clustering because it uses subsink. The subsink aggregates the data and send to the main sink.

The proposed scheme has following steps:

Step 1. Deployment of the nodes.

Step 2. Division of the network into the clusters.

Step 3. Placement of the multiple sink node in the clusters.

Step 4. Broadcasting of the hello messages by sink nodes to the cluster members.

Step 5. Formation of the route from the sink node to the destination.

Step 6. Source sends data to the respective sink nodes.

Step 7. Subsink aggregates the data and send to main sink. It continues until the last node alive.

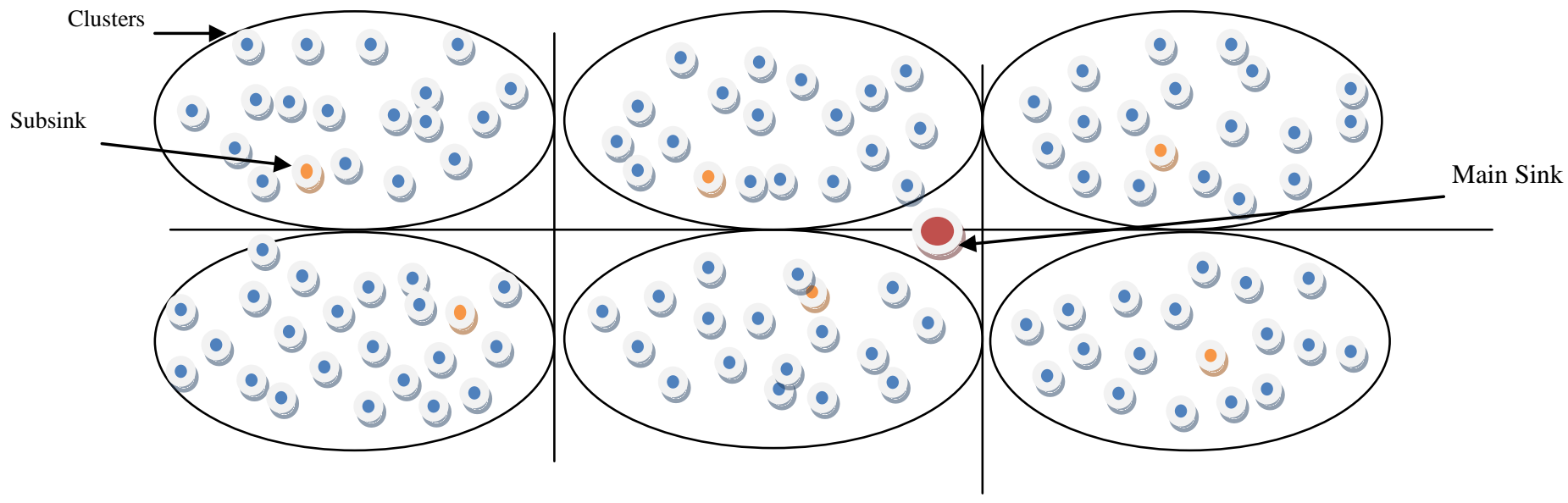

Fig 3.1: EEEGBC Network 


\section{SIMULATION AND ANALYSIS}

In this section, the proposed method has been simulated in Network Simulator 2 (NS2.35) and the simulation results are presented. The operating system is windows 7. NS2 is not supported by windows environment so VMware workstation is installed to provide the virtual environment for the installation of Ubuntu. The simulation parameters are that are used for experiment are listed in Table 1.

\begin{tabular}{|c|c|}
\hline Parameter & Value \\
\hline Simulator & NS2.35 \\
\hline Channel & Wireless Channel \\
\hline Propagation Model & 56 \\
\hline No. of nodes & Two Ray Ground \\
\hline Dimensions of Simulated Area $1000 \mathrm{~m}$ \\
\hline Queue & Drop Tail \\
\hline Antenna & Omni-Directional \\
\hline Routing Protocol & AODV \\
\hline Energy Model & 20J \\
\hline Initial Energy & \\
\hline
\end{tabular}

\subsection{PERFORMANCE METRICES}

We have three metrices to evaluate the performance of proposed scheme.

\subsubsection{Stability Period}

It is the time interval from the start of the operation until the first node death.

\subsubsection{Middle Node Death}

It is the time when half of the nodes got death.

\subsubsection{Network Lifetime}

It is the time interval from the start of the operation until the last node alive.

\section{SIMULATION RESULTS}

Following are the simulation results of proposed scheme based upon above stated performance metrics.

\subsection{Stability Period}

The Stability Period of the proposed scheme is analyzed with respect to time. The first node dies at the time $52.941788 \mathrm{~ms}$ with energy 0.275804 after the simulation starts.

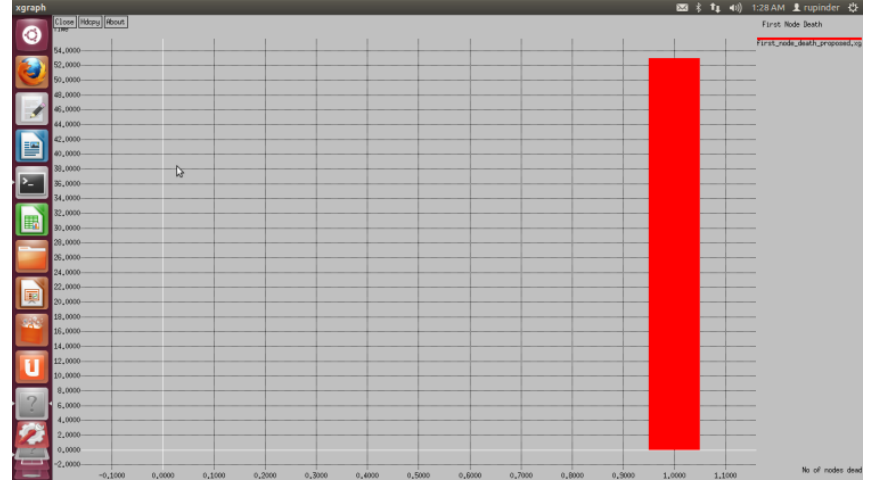

Fig 5.1 Stability Period

\subsection{Middle Node Death}

The Middle Node Death analysis of the proposed scheme is analyzed with respect to time. After the simulation starts, the first half of the nodes (21 nodes) get dies at the time $59.2166 \mathrm{~ms}$

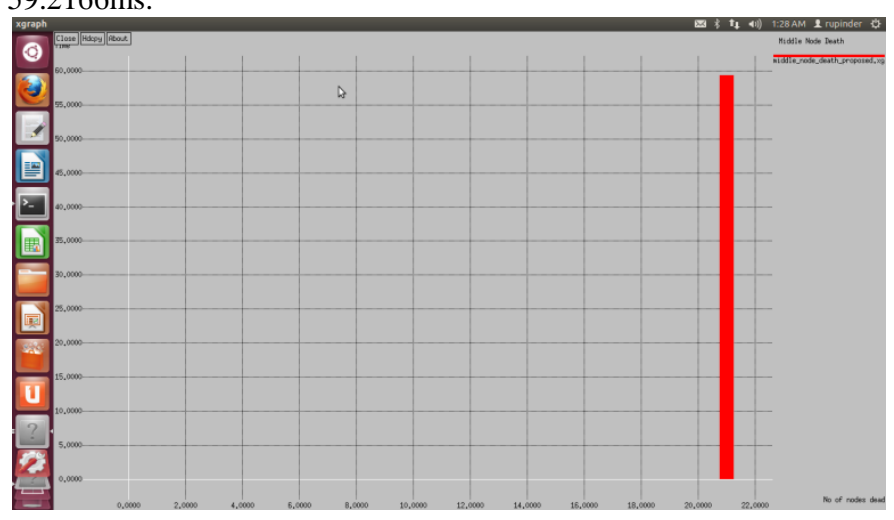

Fig 5.2 Middle Node Death

\subsection{Network Lifetime}

The Network Lifetime of the proposed scheme is analyzed with respect to time. After the simulation starts, the last node dies at the time $61.0274 \mathrm{~ms}$

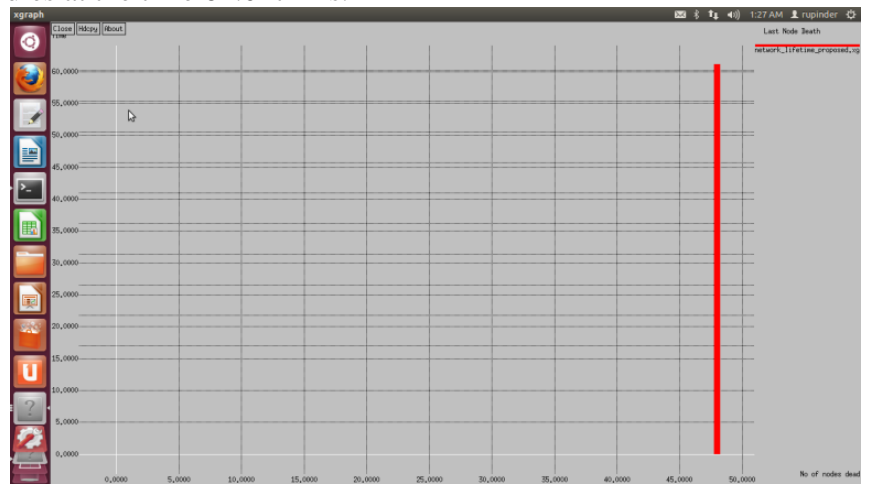

6. CONCLUSION AND FUTURE WORK

In this work we have analyzed the performance of the network under the use multiple sinks that are used for collecting the data from the network. The proposed scheme's performance calculated in terms of network lifetime, middle node death and stability period. In the future work, the above scheme can be implemented on large networks. 


\section{REFERENCES}

[1] Barra'a A. Attea, Enan A. Khalil, “A New Evolutionary Based Routing for Clustered Heterogenous Wireless Sensor Networks", Applied Soft Computing, 1950-1957, 2012.

[2] Basit Manzoor, et al. "Q-LEACH: A New Routing Protocol for WSNs." Procedia Computer Science 19,926931, 2013.

[3] B. V. Manikyala Rao, et al., "Energy Efficient Routing Protocols in Wireless Sensor Networks", IJCC, Vol.2, Issue 1, 2013.

[4] Dali Wei, et al., "An Energy-Efficient Energy Clustering Solution for Wireless Sensor Networks", IEEE Transactions on Wireless Communication, September 2011.

[5] Deepali, Padmavati, "Improved Energy Efficiency Semi Static Routing Algorithm Using Sink Mobility for WSNS”, IEEE, March 2014.

[6] Fuad Bajaber, Irfan Awan, "Adaptive Decentralized ReClustering Protocol for Wireless Sensor Networks", Journal of Computer and System Sciences, 282-292, 2011.

[7] G. Kalpana, Dr. T. Bhuvneshwari, "A Survey on Energy Efficient Routing Protocols for Wireless Sensor Networks", NCICT, 2011.

[8] H. Sivasankari, et al., "Multiple Dynamic Sinks to Maximize the Lifetime and Energy Conservation of Energy in Wireless Sensor Networks", International Journal of Computer Theory and Engineering, Feb 2012.

[9] H. Srikanth. Kamath, "Energy Efficient Routing Protocol for Wireless Sensor Networks", International Journal of Advanced Computer Research, Vol.-3, No.-2 Issue-10 June-2013.

[10] Jiguo Yu, et al., "A Cluster-Based Routing Protocol for Wireless Sensor Networks With nonuniform Node Distribution." AEU-International Journal of Electronics and Communications 66.1, 54-61, 2012.

[11] Jin Wang, at. el., "An Energy-efficient Competitive Clustering Algorithm for Wireless Sensor Networks using Mobile Sink", International Journal of Grid and Distributed Computing,Vol. 5, No. 4, December 2012.

[12] Jiguo Yo, et al., "A Cluster-Based Routing Protocol for Wireless Sensor Networks With nonuniform Node Distribution”, 2012.

[13] Mazid I. Khan, et al., "Static V/S Mobile Sink: The Influence of Basic Parameters on Energy Efficiency In Wireless Sensor Networks", Computer Communications, 965-978, 2013.

[14] Mohsin Raza Jafri, et al., "Maximizing the Lifetime of Multi-Chain PEGASIS using Sink Mobility", World Applied Science 21(9), 1283-1289, 2013.

[15] M. Emre Keskin,et al., "Wireless Sensor Network Lifetime Maximization by Optimal Sensor Deployment, Activity Scheduling, Data Routing And Sink Mobility", Ad Hoc Nwtworks,2014.

[16] M. Madheswaran, R. N. Shanmugasundaram, "Enhancements of LEACH Algorithm for Wireless Networks: A Review", Dec 2013.
[17] Md. Golam Rashed, et al., "WEP: An Energy Efficient Protocol for Cluster Based Heterogeneous Wireless Sensor Network" International Journal of Distributed and Parallel Systems, Vol.2, No.2, March 2011.

[17] Miguel Garcia, et al. "Saving Energy and Improving Communications using Cooperative Group-Based Wireless Sensor Networks." Telecommunication Systems 52.4, 2489-2502, 2013.

[18] Meenakshi Diwakar, Sushil Kumar, “An Energy Efficient Level based Clustering Routing Protocol for Wireless Sensor Networks", International Journal Of Advanced Smart Sensor Network Systems, Vol 2, No. 2,April 2012.

[19] Nadeem Javaid, et al., "EDDEEC: Enhanced Developed Distributed Energy-Efficient Clustering for Heterogeneous Wireless Sensor Networks." Procedia Computer Science 19 914-919, 2013.

[20] Nagaraj, C. N. Chinnaswami, "Dynamic Routing for ADA in Wireless Sensor Networks", IJCSMC, 2014.

[21] Nauman Aslam, et al., "A Multi-Criterion Optimization Technique for Energy Efficient Cluster Formation in Wireless Sensor Networks." Information Fusion 12.3, 202-212, 2011.

[22] Neha Upadhayay, Vikram Jain, "Node and Sink Mobility Supported Routing Protocol in Wireless Sensor Network with Improved Energy Efficiency”, IJCSMC, June 2013.

[23] Nikolaos A. Pantazis, Stefanos A. Nikolidakis, and Dimitrios D. Vergados. "Energy-Efficient Routing Protocols In Wireless Sensor Networks: A Survey." Communications Surveys \& Tutorials, IEEE 15.2, 551$591,2013$.

[24] Roshani Kushavaha, Umesh kumar Chourasiya, Mahesh Gour, "A study of Power and Energy Aware Routing Technologies in Wireless Sensor Network", International Journal of Computer Science and Information Technologies, Vol. 5, 2014.

[25] Samer AB Awwad, et al., "Cluster Based Routing Protocol for Mobile Nodes In Wireless Sensor Network." Wireless Personal Communications 61.2, 251-281,2011. [26] S. A. Ahson Rajon, Md. Mahbubur Rahman, "Energy Efficient Routing in Wireless Sensor Network", IJCIT 2012

[27] Stefanos A. Nikolidakis, et al., "Energy Efficient Routing in Wireless Sensor Networks through Balanced Clustering”, ISSN 2013.

[28] Sudhanshu Tyagi,Neeraj Kumar, "A Systematic Review on Clustering And Routing Techniques based upon LEACH Protocol for Wireless Sensor Networks", Journal of Network and Computer Applications,623-645, 2013.

[29] V. A. Amala Shiny, V. Nagaranjan, "Energy Efficient Routing Protocol for Mobile Wireless Sensor Networks", International Journal of Computer Application, April 2012. 